International Journal of Biomedicine | June 2021 - Volume 11, Issue Suppl_1: Abstracts from the Third Russian International Conference "Cryo-electron microscopy 2021: achievements and prospects"

POSTER ABSTRACT PRESENTATIONS

SESSION TITLE: STRUCTURE OF MEMBRANE PROTEINS

DOI: 10.21103/IJBM.11.Suppl_1.P14

\title{
Abstract P-14: Molecular Modeling of the Transmembrane Domain of the SARS Cov-2 S-Protein and its Interaction with the Membrane
}

Valery Novoseletsky, Marine Bozdaganyan, Daniil Litvinov, Olga Sokolova M. V. Lomonosov Moscow State University, Moscow, Russia

Background: The spike glycoprotein of SARS-coronavirus mediates the early events leading to infection of cells, including fusion of the viral and cellular membranes. The spike is a type I membrane glycoprotein that possesses a conserved transmembrane anchor and an unusual cysteine-rich domain that bridges the putative junction of the anchor and the cytoplasmic tail. In this study, we examined the role of these carboxyl-terminal domains in S-protein interaction with membrane.

Methods: Structural model of the trimeric TM domain and adjacent fragments of ecto- and endo domains (residues 1157-1256) of the S-protein was built by homology basing on the solution structure of the SARS-coronavirus S-protein HR2 domain (pdb-code 2fxp), the structure of the transmembrane domain of HIV-1 gp41 in bicelle (5jyn), and assumption of generally coiled-coil fold of the considered domain. C-terminus of the domain was left unstructured but fully palmitoylated. Molecular dynamics simulation in heterogeneous lipid bilayer was prepared with CHARMM-GUI and performed with Gromacs during $100 \mathrm{~ns}$. Results: 1. Ectodomain fragment (residues 1157-1212) demonstrates a tilt by the angle of 40-60 degrees from the axis of the TM domain (residues 12131237). This tilt is facilitated by glycine residues in position 1204. 2. Cholesterol molecules of the bottom layer tend to localize around protein due to interaction with palmitoyl tails while lipids in the upper layer do not show such tendency.

Conclusion: Performed molecular simulations show that both palmitoylation and a large cluster of aromatic residues provide high stability of the S-protein TM domain.

Key Words: SARS Cov-2 $\bullet$ S-protein $\bullet$ palmitoylation $・$ molecular modeling 
This work was supported by the Russian Foundation for Basic Research (Grant No. 20-04-60258)

*Corresponding author: Valery Novoseletsky. E-mail:valery.novoseletsky@yandex.ru

International Journal of Biomedicine. 2021;11 Suppl 1: S17.

doi: 10.21103/IJBM.11.Suppl_1.P14

(C)2021 International Medical Research and Development Corporation 